\title{
A brief insight into the role of image fusion in oncology.
}

\author{
Ayush Dogra $^{\text {** }}$, Bhawna Goyal ${ }^{1}$, Sunil Agrawal ${ }^{1}$, Ebenezer Daniel ${ }^{2}$ \\ ${ }^{1}$ UIET, Panjab University, Chandigarh, India \\ ${ }^{2}$ Vignan's Foundation for Science, Technology \& Research, Andhra Pradesh, India
}

\begin{abstract}
Medical imaging has emerged as an indispensable tool in the evaluation, treatment and planning of the cancerous ailments. Recently the imaging modalities acquired by various sensors for instance SPECT/CT and PET/CT is readily fused together for the precise visualization of the various kinds of tumours and cancerous cells. In this write up we aim to present a brief insight into the significance and relevance of various fused imaging modality systems in the diagnosis and treatment of patients with cancer.
\end{abstract}

Keywords: Oncology, Fusion, CT, PET, SPECT, tumour, cancer.

Accepted on 14 March 2018

\section{Introduction}

It is imperative in medical imaging that different imaging technologies provide different types of information. These different types of information have to be mentally integrated by the radiologist to draw some useful conclusion. In case of medical visual perception a radiologist must switch between sensors to observe one sensor modality at a time. This makes it difficult in identifying the interactions among patterns. Fusion may provide the means for an observer to perceive all the available visual information at a single glance, in correct registration [1,2]. The medical image fusion is a significant imaging technology which helps in integrating the divergent multi-sensory information for the ailment under study in a single composite image. The CT images enable the visualization of the morphological structures and the anatomical features of the subject under study. However they are less sensitive to the functional details. Single-photon emission computed tomography (SPECT) and positron emission tomography (PET) are precise visualization modes for detecting cancer related metabolic abnormalities; however they tend to fail while presenting the anatomical structures. Image fusion provides a trivial solution by combining these modalities while overcoming the drawbacks of both the modalities. The fusion of the anatomical details in CT and molecular functionality in PET/SPECT has immeasurably facilitated the identification of cancer stage, planning and treatment. The radioactive isotope is injected into the patient body for PET scanning. The glucose component of the FDG (fluoro-2-deoxyD-glucose) allows it to reach the sugar seeking cancerous cells. Hence a disproportionate amount of FDG is localized amongst the malignancies, enabling the visualization of tiny tumors on PET scans. The ultimate outcome of the fusion of these modalities results in the projection of the anatomical structural details over the functional image to localize cancerous tumors. The PET/CT fusion is one of the major fusion methods which are readily utilized these days in the field of oncology [3].

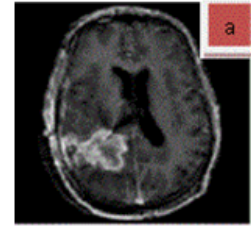

MR Image

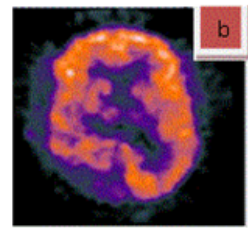

PET Scan

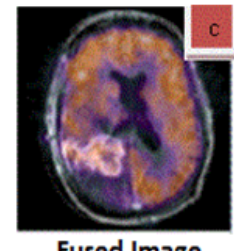

Fused Image
Figure 1. MR/PET fusion system

Besides these some of the other imaging modalities are fused together which finds application in the medical oncology [4,5]. For instance a research study given in [6] pre-biopsy magnetic resonance imaging is fused with trans-rectal ultrasound imaging to identify and biopsy lesions for prostate cancer. Due to the higher soft tissue contrast of MRI, the CT images are often replaced with MRI images so that the tumour volume can often be defined with better accuracy in brain. Figure1 shows the MRI-PET fusion system which is readily employed for the clinical diagnosis and treatment procedure of sarcoma, cerebral toxoplasmosis and astrocytoma. Furthermore studies given in [7] validate the gross tumour volume (GTV) delineation CTMRI fused image data set as compared to CT alone. The basic mechanism of image fusion using taking the average of the pixel intensities of the two source images and inserting into the fused image.

Fused IMG $=\sum_{i=0}^{N} \frac{\left(S_{i j}\right)+\left(T_{i j}\right)}{2}$

S(ij) and T(ij) are the pixel intensities of the source images. There are several representative image fusion algorithms available in literature which transfer the most of the edge information from the source images to the fused images which help the radiologists to arrive at the accurate diagnosis. However in some cases the inefficient image fusion algorithms create halo and gradient reversal artefacts which lead to false diagnosis and false positivity. Flowchart of image fusion phenomenon is depicted in figure 2 . 


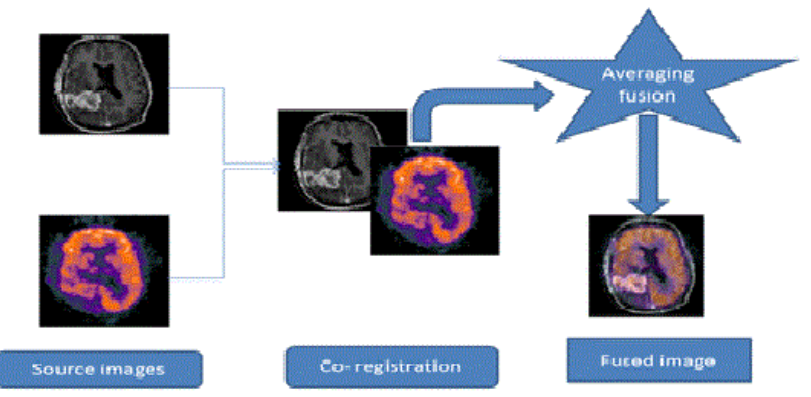

Figure 2. Depicts basic methodology of image fusion

Henceforth both software-based fusion systems and dualmodality integrating hardware systems are best suited for improving diagnostic accuracy and treatment planning in oncological applications $[8,9]$.

\section{References}

1. Dogra A, Goyal B, Agrawal S. Osseous and digital subtraction angiography image fusion via various enhancement schemes and Laplacian pyramid transformations. Future Gener Comput Syst. 2018;82:149-57.

2. Dogra A, Goyal B, Agrawal S, et al. Efficient fusion of osseous and vascular details in wavelet domain. Pattern Recognition Letters. 2017;15:189-93.

3. Dogra A, Goyal B, Agrawal S. From multi-scale decomposition to non-multi-scale decomposition methods: A comprehensive survey of image fusion techniques and its applications. IEEE Access. 2017;5:16040-67.
4. Daniel E, Anitha J, Kamaleshwaran K, et al. Optimum spectrum mask based medical image fusion using Gray Wolf Optimization. Biomed Signal Process Control. 2017;34:36-43.

5. Paulino AC, Thorstad WL, Fox T. Role of fusion in radiotherapy treatment planning. Semin Nucl Med. 2003;33:238-43.

6. Pinto PA, Chung PH, Rastinehad AR, et al. Magnetic resonance imaging/ultrasound fusion guided prostate biopsy improves cancer detection following transrectal ultrasound biopsy and correlates with multiparametric magnetic resonance imaging. J Urol. 2011;4:1281-5.

7. Iqbal K, Altaf S, Aqeel M, et al. CT-MRI Image Fusion for Accurate Delineation of Gross Tumor Volume in Three Dimensional Conformal Radiation Therapy. J Radiol. 2015;4:2.

8. Schillaci O, Simonetti G. Fusion imaging in nuclear medicine-applications of dual-modality systems in oncology. Cancer Biother Radiopharm. 2004;1:1.

9. Dwork N, Eric L, John P, et al. "Formulation of image fusion as a constrained least squares optimization problem. J Med Imaging. 2017;4:014003.

\section{*Correspondence to:}

Ayush Dogra,

UIET, Panjab University,

Chandigarh, India

E-mail: ayush123456789@gmail.com 